\title{
EL JUEGO COOPERATIVO COMO MÉTODO PARA FAVORECER LA INCLUSIÓN Y EL DESARROLLO DE CONDUCTAS PROSOCIALES
}

Cooperative play as a method to promote inclusion and development of prosocial behavior

O jogo cooperativo como um método para promover a inclusão e o desenvolvimento de comportamentos pró-sociais

Ana $\mathbf{M}^{\mathrm{a}}$ Mega Avellaneda (1)

Marta Liesa Orús (2)

(1) Facultad de Ciencias Humanas y de la Educación de Huesca, Universidad de Zaragoza. Correo electrónico: anahuesketa@hotmail.com

(2) Universidad de Zaragoza, España. Correo electrónico: martali@unizar.es

\begin{abstract}
Resumen
Esta comunicación presenta un estudio que se ha llevado a cabo con en niños/as de Educación Primaria de dos centros educativos públicos de Huesca; C.E.I.P. Pio XII y C.E.I.P. Pedro J. Rubio. La intención es explorar cuáles son los efectos de un proyecto de aprendizaje cooperativo desarrollado en los recreos de dos centros de Educación Infantil y Primariadiferenciados por el nivel socio-cultural de la población. Se pretende conocer silos valores emocionales que subyacen de la práctica lúdica contribuyen a eliminar situaciones de exclusión social, y si los efectos del juego cooperativo, difieren o no, entre dos realidades que representan a la diversidad de la ciudad. Los resultados manifiestan como el alumnado, independientemente del contexto del que proviene, mejora sus habilidades intra e interpersonales, y cómo se generan relaciones educativas más positivas.
\end{abstract}

Palabras clave: Conductas prosociales; inclusión; juego cooperativo; comunicación

\begin{abstract}
This communication presents a study that has been carried out with children of Primary Education in two state schools in Huesca; C.E.I.P. Pio XII and C.E.I.P. Pedro J. Rubio. The intention is to explore what effects of a cooperative learning project are. The aim is


to discover whether learning resulting is differentiated by the socio-cultural level of the population, and if emotional values of playing contribute to eliminate situations of social exclusión of two realities that represent the diversity of the city. The results show how students, regardless of the context that comes, improve their intra and interpersonal skills, and how cooperative gamescause more positive educational relationships.

Keywords: Prosocial behavior; inclusion; cooperative play; Communication

\section{Resumo}

Este trabalho apresenta um estudo foi realizado em crianças / Educação Primária como duas escolas públicas de Huesca; C.E.I.P. Pio XII e C.E.I.P. Pedro J. Rubio. A intenção é explorar o que os efeitos de um projeto de aprendizagem colaborativo desenvolvido nas recriações duas pré-escolas e primários diferenciados por nível sócio-cultural da população são. Ele pretende saber se os valores emocionais que estão na base da ajuda prática lúdica eliminar situações de exclusão social, e se os efeitos de jogo cooperativo, diferem ou não, entre duas realidades que representam a diversidade da cidade. Os resultados se manifestam como os alunos, independentemente do contexto a partir do qual se trata, melhora suas habilidades intra e interpessoais, e como as relações educacionais mais positivos são gerados.

Palavras-chave: Comportamento pró-social; inclusão; jogo cooperativo; Comunicação

\section{Introducción}

Actualmente vivimos en una sociedad a la que se le reconoce cierto prestigio por su intento de crear escuelas inclusivas, donde todo alumno es aceptado independientemente de sus características (Dueñas, 2010; Leiva, 2008).

Según Camacho (2013) o López (2008) las "herramientas sociales" no son innatas al ser humano, y desde esta perspectiva se interpreta que el juego cooperativo podría contribuir al desarrollo de habilidades prosociales (Álvarez, 2011; Bargas, Briceño \& Loria, 2016), y consecuentemente en la prevención de la exclusión social y el acoso escolar. Estos estudiosos argumentan que la cooperación impregna la práctica de conductas asertivas por su papel trascendental en el desarrollo emocional, social y afectivo. En esta línea, podemos pensar que la cooperación podría ser una estrategia inclusiva, contraria a la competitividad, a desarrollar en los recreos, y encaminada a El juego cooperativo como método para favorecer la inclusión y el desarrollo de conductas prosociales 
educar bajo la ayuda incondicional, la solidaridad entre los iguales y la aceptación (Pujolás, 2003). Nuestro estudio pretende valorar si el aprendizaje resultante de las interacciones cooperativas difiere, o no, de un contexto educativo a otro, si sirve para evitar situaciones conflictivas durante el recreo escolar, y si mejora la discriminación de género entre chicas y chicos en los momentos de juegoen ambos centros.

\section{Contexto del estudio. Participantes}

La unidad de análisis corresponde al proyecto de "Recreos Cooperativos e Inclusivos", una experiencia de la Facultad de Ciencias Humanas y de la Educación de Huesca. En el estudio participan dos centros públicos de Huesca con realidades sociales y culturales diferentes entre sí que representan, en buena parte, a la diversidad presente en nuestra ciudad.

Por un lado, el alumnado de $3^{\circ}$ y $4^{\circ}$ de E.P del C.E.I.P. Pedro J. Rubio; un colegio deseado por las familias, con un exceso de demanda y una elevada ratio, situado en un entorno sociodemográfico con estabilidad laboral, y un nivel socioeconómico medio-alto. Por otro lado, el alumnado de $1^{\circ}$ y $2^{\circ}$ de E.P del C.E.I.P. Pio XII; un contexto que aglutina a gran parte de la población en riesgo de exclusión social y en situaciones desfavorables; mayoritariamente población de etnia gitana y/o de origen inmigrante, y cuenta con dos aulas de Educación Especial.

La elección de la muestra se ha confeccionado a partir de la decisión que toma el equipo directivo de ambos centros, seleccionando estos cursos y no otros, por la presencia en ellos, de grupos sociales en situaciones de riesgo social o exclusión. En ambos centros educativos encontramos alumnado asilado durante el recreo, la aparición de conductas agresivas y/o disruptivas, algún grupo cultural minoritario, e incluso algún caso de acoso escolar).

\section{Método y procedimiento}

Se trata de un estudio descriptivo que muestrala eficacia del juego cooperativo desde la perspectiva y la opinión de los participantes en los “Recreos Cooperativos”.

Los datos se recogen de la percepción de los estudiantes de Magisterio que están haciendo prácticas escolares en cada centro y que observan al alumnado. Semanalmente planifican y dirigen los juegos, y recogen información marcando la aparición de unas determinadas pautas comportamentales recogidas en el registro. 
Como técnicas de investigación se utilizan un registro observacional y un cuestionario, y se realiza una prueba piloto en la cual participa una pequeña muestra de 4 alumnos elegidos al azar de entre el grupo de la muestra participante.

El registro consta de una valoración del comportamiento del alumnado, de los juegos planificados y del profesorado, y el cuestionario consta de siete ítems de tipo Likert de respuestas alternativas relacionados con la dimensión social y la relación con el juego cooperativo.

\section{Resultados y discusión}

A pesar de que en el C.E.I.P. Pio XII surgen más conflictos que en el C.E.I.P. Pedro J. Rubio (52,7\% frente a un 5,3\%), son más participativos en los juegos cooperativos (83,3\% frente a un $45 \%$ ) y se muestran menos retraídos a la hora de interaccionar (11,2 \% frente a un 20\%). Sin embargo, presentan una menor capacidad en la resolución de los conflictos que surgen durante los recreos (5,5 \% frente a un 26,6\%).

En las sesiones iniciales se observa la presencia de actitudes de desconfianza, y relaciones intergrupales estereotipadas, comportamientos de burla, indiferencia y ridiculización, en ambos centros educativos, aspectos que varían positivamente. En la primera sesión, el 53 \% del alumnado del C.E.I.P. Pío XII y el 22.22 \% del alumnado del C.E.I.P. Pedro J. Rubio temían las posibles represalias si su equipo fracasaba por su culpa. Sin embargo, estos comportamientos competitivos se van convirtiendo en cooperativos conforme transcurren las sesiones. Así, un 88,3 \% del alumnado del C.E.I.P. Pío XII, frente aun 57,14 \% del alumnado del C.E.I.P. Pedro J. Rubio consideran que “todos son necesarios para lograr el éxito”.

Se observa cómo el centro con mayores rivalidades culturales obtiene mejores puntuaciones e incluso aparecen, con mayor frecuencia, gestos de apoyo y de ánimo recíprocos. Las valoraciones finales indican que se sienten más aceptados y con menor temor a las expectativas ajenas que les impiden relacionarse con total libertad

\section{Conclusiones}

Los resultados desmienten que las condiciones socioculturales adversas no impiden el logro de una educación de calidad. El juego ha mejorado la autoestima y el autoconcepto del alumno según la valoración que proporciona el propio alumnado. 
Se pretendía analizar el proyecto de "Recreos Cooperativos e Inclusivos”, desde la valoración del alumnado partícipe. El propio alumnado considera que los juegos han servido para disminuir los conflictos y mejorar los lazos afectivos entre niños de índole étnica diversa.

Si los conflictos sociales y las actitudes de desconfianza, se gestionan correctamente y no son vistos como un problema sino como una oportunidad, podemos lograr el cambio de actitudes cuando las escuelas indaguen en la cultura del diálogo y la cooperación (Ainscow, 2012; Arnaiz, 2012; Echeita \& Ainscow, 2011; Melero, 2011).

\section{Referencias}

Ainscow, M. (2012). Haciendo que las escuelas sean más inclusivas: lecciones a partir del análisis de la investigación internacional. Revista de Educación inclusiva, 5(1), 39-49.

Álvarez, D. G. (2011). La Inteligencia Emocional: competencias esenciales para la vida ciudadana. REDIELUZ, 1(1), 72-79.

Arnaiz, P. (2012). Escuelas eficaces e inclusivas: cómo favorecer su desarrollo. Educatio siglo XXI, 30(1), 25-44.

Bargas, R. M. O., Briceño, E. D., \& Loria, M. D. (2016). Juegos cooperativos: efectos en el comportamiento asertivo en niños de 6o. grado de escuelas públicas. Revista Electrónica de Investigación Educativa, 18(3).

Camacho, L.J. (2013). El juego cooperativo como promotor de habilidades sociales en niñas de 5 años. Perú: Pontificia Universidad Católica del Perú.

Dueñas, M. ${ }^{\text {a }}$ L. (2010). Educación inclusiva. REOP, 21(2), 358-366.

Echeita, G., \& Ainscow, M. (2011). La educación inclusiva como derecho: marco de referencia y pautas de acción para el desarrollo de una revolución pendiente. Tejuelo, 12, 26-45.

Leiva, J. (2008). Interculturalidad, gestión de la convivencia y diversidad cultural en la escuela: un estudio de las actitudes del profesorado. Revista Iberoamericana de Educación, 46, 1-14. 
López, M. (2008). La integración de las habilidades sociales en la escuela como estrategia para la salud emocional. Psicología sin fronteras: revista electrónica de intervención psicosocial y psicología comunitaria, 3(1), 16-19.

Melero, M. (2011). Barreras que impiden la escuela inclusiva y algunas estrategias para construir una escuela sin exclusiones. Innovación Educativa, 21, 37-54

Pujolás, P. (2003). Aulas inclusivas y aprendizaje cooperativo. Barcelona: Universidad de Vic. 